and survival analysis, were used to investigate associations between hip shape modes and change in cartilage volume, incident BMLs, worsening knee pain and left KR respectively. All models were adjusted for baseline age, sex, BMI, knee injury or surgery and hip radiographic osteoarthritis(ROA), while the KR model was additionally adjusted for WOMAC pain and knee ROA.

Results: Ten hip shape modes were identified, describing $78 \%$ of the total shape variance in descending order from mode 01 (31\% variance) to mode $10(1.82 \%$ variance). Hip shapes with a larger greater trochanter (mode 07$)$ were associated with lower knee cartilage volume loss (Beta:2.14, 95\% Cl:0.07,4.21), while a shorter and narrower femoral neck shape (mode 09) was related to increased volume loss (Beta:-3.86, 95\% Cl:-6.16,-1.56). Increasingly non-spherical femoral head (mode 04) was associated with an increased risk of incident BMLs (RR:1.19, $95 \% \mathrm{Cl}: 1.07,1.34)$. Those with a longer, wider femoral neck and a larger femoral head (mode 01) had an increased risk of worsening knee pain (RR:1.33, $95 \% \mathrm{Cl}: 1.09,1.61$ ), whereas those with a smooth curving upper femoral neck (mode 09) had a lower risk of worsening knee pain (RR:0.78, 95\% Cl:0.67,0.90). A larger greater trochanter and wider femoral neck shape (mode 08) was associated with an increased risk of $\mathrm{KR}$ (RR:1.73, 95\% Cl:1.18,2.52), while increasing acetabular coverage (mode 10) was associated with a lower risk of KR (RR:0.54, $95 \% \mathrm{Cl}: 0.36,0.8)$.

Conclusions: Hip shape variations were associated with significant MRI-based and clinical outcomes in knee over 10.7 years, possibly due to biomechanical, lifestyle or other factors related to both joints. These results suggest that hip shape may play an important role in the onset and progression of knee osteoarthritis over time.

Disclosure of Interest: None declared

DOI: 10.1136/annrheumdis-2018-eular.4985

\section{SAT0563 IDENTIFICATION AND VALIDATION OF PHYSICAL ACTIVITY PHENOTYPES FOR KNEE OSTEOARTHRITIS: A POPULATION-BASED COHORT STUDY}

I.P. Munugoda ${ }^{1}$, F. Pan ${ }^{1}$, K. Wills ${ }^{1}$, F. Cicuttini ${ }^{2}$, S.E. Graves ${ }^{3}$, M. Lorimer ${ }^{4}$, G. Jones ${ }^{1}$, M.L. Callisaya ${ }^{1}$, D. Aitken ${ }^{1} .^{1}$ Menzies Institute for Medical Research, University of Tasmania, Hobart, Tasmania; ${ }^{2}$ Department of Epidemiology and Preventive Medicine, Monash University Medical School, Melbourne, Victoria; ${ }^{3}$ Australian Orthopaedic Association National Joint Replacement Registry (AOANJRR); ${ }^{4}$ South Australian Health and Medical Research Institute (SAHMRI), Adelaide, South Australia, Australia

Background: The identification of phenotypes to reduce heterogeneity of characteristics is important in understanding the development and progression of knee osteoarthritis.

Objectives: This study aimed to identify physical activity (PA) phenotypes and to investigate the association of these phenotypes with tibial cartilage volume, bone marrow lesions (BMLs) and knee replacements (KR) over 10.7 years.

Methods: 1046 community-dwelling older adults aged $50-80$ years were studied. At baseline, PA was measured by pedometers (steps/day), knee pain was assessed using Western Ontario and McMaster Universities Osteoarthritis Index (WOMAC) and body mass index (BMI) was determined utilising objective weight and height measures. MRI scans were conducted at baseline and 10.7 years to assess tibial cartilage volume and BMLs. The incidence of KR was determined by data linkage to the Australian Orthopaedic Association National Joint Replacement Registry. Latent class analysis was used to determine 'PA phenotypes' based on PA, BMI and WOMAC pain at baseline. Linear mixed-effects models and log-binomial models were used to estimate the associations between the identified PA phenotypes with change in cartilage volume, incident BMLs, and KR surgery over 10.7 years. All models were adjusted for age, sex, and history of knee injury or surgery, while the KR model was additionally adjusted for the prevalence of knee radiographic osteoarthritis.

Results: Three PA phenotypes were identified: Class 1: Normal/overweight participants with low levels of PA and low pain (42\%); Class 2: Obese participants with low levels of PA and high pain (26\%); Class 3: Normal/overweight participants with high levels of PA and low pain (32\%). Mean cartilage volume loss over 10.7 years was $465 \pm 231 \mathrm{~mm}^{3} .221$ participants had an incident BML while 74 had an incident KR. Class 2 participants had greater cartilage volume loss over 10.7 years (Beta: $-79.9,95 \% \mathrm{Cl}-135.8,-23.9)$ and, had a higher risk of KR (RR 2.36, $95 \% \mathrm{Cl} 1.20,4.67)$ compared to Class 1 participants. Class 3 was not associated with cartilage volume loss (Beta: $-36.2,95 \% \mathrm{Cl}-86.6,14.1)$ or risk of $\mathrm{KR}$ (RR $0.86,95 \% \mathrm{Cl} 0.40,1.84$ ) compared to Class 1 . Similarly, PA phenotypes were not associated with incident BMLs (Class 2: RR 1.15, 95\% Cl 0.88, 1.50; Class 3: RR $1.19,95 \% \mathrm{Cl} 0.93,1.54$; compared to Class 1 ).

Conclusions: Our findings support the existence of homogeneous PA profiles, and suggest that PA interacts with body weight and knee pain and has long-term impacts on osteoarthritis outcomes.

Disclosure of Interest: None declared

DOI: 10.1136/annrheumdis-2018-eular.3197

\section{SAT0564 EFFECTS OF EDUCATION AND INCOME ON PREVALENCE, INCIDENCE, AND PROGRESSION OF RADIOGRAPHIC KNEE OSTEOARTHRITIS: AN} ANALYSIS OF THE OSTEOARTHRITIS INITIATIVE DATA

I. Shirinsky, V. Shirinsky. Research Institute Of Fundamental And Clinical Immunology, Novosibirsk, Russian Federation

Background: Low socioeconomic status (SES) is one of the strongest predictors of morbidity and mortality from many chronic diseases including cardiovascular diseases, obesity, and diabetes. ${ }^{1}$ There is insufficient data regarding impact of SES on knee osteoarthritis (OA)

Objectives: To evaluate the associations between education, income levels and prevalence, incidence, and progression of radiographic knee $\mathrm{OA}$

Methods: For the current analysis we used data from the publically available Osteoarthritis Initiative (OAI) database. The education status of the participants was dichotomized into either low/moderate or high educational attainment. The income status was dichotomized using $50 \mathrm{~K}$ US threshold. A baseline sample was used to analyse the impact of SES on knee OA prevalence (prevalence sample). To evaluate the effects of SES on knee OA incidence and progression we analysed the samples of $\mathrm{OAI}$ participants with $\mathrm{KL}<2$ at baseline (incidence sample) and OAI participants with $\mathrm{JSN}<3$ at baseline (progression sample), respectively. We used logistic regression models to assess the association between SES and prevalence and incidence of radiographic knee OA (defined as KL2 and JSN or joint replacement), and disease progression (defined as increase in semiquantitative JSN or a new knee replacement). Generalised estimating equations (GEE) were used to adjust for the correlation between knees. The models were adjusted for multiple covariates including age, race, and body mass index.

Results: Prevalence, incidence, and progression samples consisted of 4371 participants (8741 knees), 2268 participants (4535 knees), and 3950 participants (4013 knees), respectively.

Higher education attainment and higher income were associated with decreased prevalence of the knee OA in the crude analyses. After adjustment for confounders these associations became insignificant (Table). There was no effect of SES on incidence and progression of the knee OA.

Table

\begin{tabular}{lcccc}
\hline & \multicolumn{4}{c}{ Prevalence } \\
\cline { 2 - 5 } & Unadjusted & & \multicolumn{2}{c}{ Adjusted } \\
\cline { 2 - 5 } & OR $(95 \% \mathrm{Cl})$ & P value & OR $(95 \% \mathrm{Cl})$ & $\begin{array}{c}\mathrm{P} \\
\text { value }\end{array}$ \\
\hline Education & $0.8(07-0,9)$ & $<0.001$ & $1,05(0,9-$ & 0.48 \\
Income & $0.71(0,64-$ & $<0.001$ & $1,18)$ & 0.97 \\
& $0,8)$ & & $1(0.87-1.4)$ & \\
\hline
\end{tabular}

$\mathrm{OR}$ - odds ratio, $\mathrm{Cl}$ - confidence interval

Conclusions: Higher levels of education and income are linked with decreased prevalence of radiographic knee OA. Lack of this association after adjustment suggests confounding or mediating effects of other risk factors. Future studies are needed to delineate the precise mechanisms of how SES impact knee OA.

\section{REFERENCE:}

[1] Braveman P, Gottlieb L. The social determinants of health: it's time to consider the causes of the causes. Public Health Rep 2014 Jan-Feb;129 (Suppl 2):19-31.

Disclosure of Interest: None declared DOI: 10.1136/annrheumdis-2018-eular.3424

\section{SAT0565 ADJUSTING FOR THE INTRA-ARTICULAR PLACEBO EFFECT IN KNEE OSTEOARTHRITIS THERAPIES}

I. Simsek', T. Phalen', A. Bedenbaugh', M. Phillips ${ }^{2}$, J. Tambiah' ${ }^{1}$. ${ }^{1}$ Samumed, LLC, San Diego, USA; ${ }^{2}$ McMaster University, Ontario, Canada

Background: Currently, there is a large debate regarding the appropriateness of intra-articular (IA)-saline injection as a "placebo" comparator in knee osteoarthritis $(\mathrm{OA})$ trials and meta-analyses. There is substantial evidence to suggest that the injection of saline into the joint is not without treatment effect.

Objectives: This study aimed to assess the current literature's estimates of the IA-saline treatment effect against a range of appropriate minimal clinically important difference (MCID) values to identify if IA-saline provides a therapeutic effect that is not indicative of a null-effect.

Methods: The treatment effect estimates of IA-saline and topical placebo for knee OA pain, relative to oral placebo, were derived from a published network meta-analysis Bannuru et al, 2015 and compared across a range of plausible 